\title{
Multiseptate Gallbladder with Recurrent Abdominal Pain. A Case Report and Literature Review.
}

\author{
Vidi Demko1, Xhesika Xhetani²
}

https://doi.org/10.32391/ajtes.v3i2.57

\begin{abstract}
Background: Chronic abdominal pain is a common disorder in children and adolescents worldwide. In attacking this problem, the pediatrician often concentrates on the gastrointestinal or genitourinary tract as a source of the pain. Too little attention is paid to the gallbladder as the cause of pain in this age group. ${ }^{1}$ We report a 6-year-old girl who presented with recurrent abdominal pain and was diagnosed as having a multiseptate gallbladder (MSG). MSG, although rare, should be considered in the differential diagnosis of patients presenting with recurrent abdominal pain and abdominal ultrasonography should form part of the investigation.

Keywords: gallbladder, abdominal pain, ultrasonography.
\end{abstract}

*Corresponding Author: Xhesika Xhetani

$=凶 E$-mail: xhesikaxhetani@outlook.com

${ }^{1}$ Radiology Department, University Hospital of Trauma, Tirana, Albania.

${ }^{2}$ Radiology Department, University Hospital Center "Mother Teresa", Tirana, Albania. 


\section{Full Text}

\section{Introduction}

The multiseptate gallbladder is one of the rarest congenital malformations of the gallbladder. The first detailed description of a multiseptate gallbladder appeared in the radiologic literature in 1963. ${ }^{2}$ The presenting features include abdominal symptoms such as right upper abdominal pain, nausea, and vomiting. The patients with MSG may also be asymptomatic and can be diagnosed incidentally during ultrasonography (USG) examination. In this case presentation, we aimed to present a patient with a multiseptate gallbladder who presented with recurrent abdominal pain.

\section{Case report}

A 6-year-old girl with recurrent abdominal pain of about 6 months duration was admitted to our hospital. Starting as a dull ache in the right upper quadrant of the abdomen, with no radiation to the back, the right shoulder, and the interscapular area, the pain persisted, its intensity varying from mild to moderate. There were no associated fever, nausea, vomiting, itching, or jaundice. The physical examination and laboratory studies such as complete blood count, liver function tests, electrolytes, and urinalysis revealed no specific abnormality. The sonographic examination of the abdomen was performed with a $3.5-\mathrm{MHz}$ convex transducer and a $7.5 \mathrm{-MHz}$ linear transducer. USG examination performed in supine and left decubitus posture with full inspiration. The intercostal approach was performed when necessary. On sonographic examination, the gallbladder showed multiple linear echoes, consistent with septa projecting into the lumen, some of which crossed the lumen and connected to the opposing walls mimicking a honey-comb pattern. There was no evidence of gallbladder wall thickening, gallstones or dilatation of the bile ducts. Based on these USG findings, the diagnosis of a multiseptate gallbladder was made. The patient was referred to the gastroenterology clinic.

\section{Discussion}

The multiseptate gallbladder is thought to be a congenital malformation, although the embryogenetic mechanism is not clear. Simon and Tandon proposed that, this was due to incomplete vacuolation of the developing gallbladder. Bhagavan et al. ${ }^{3}$ have suggested that, MSG may be a result of the solid embryonic gallbladder growing faster than its bed and investing peritoneum, causing aberrant bends and kinks. The same authors also postulated that, a variation in the wrinkling, lobulation, and clefting of the gallbladder (seen in cat and guinea pig embryos) may result in MSG $^{3,4}$. Asymptomatic patients are very rare in the literature ${ }^{5}$. Clinically, most patients present with colicky pain suggestive of cholecystitis, usually in the right upper quadrant or in the epigastrium, or long term abdominal symptoms such as right upper quadrant tenderness, recurrent abdominal pain, nausea and vomiting, and gastrointestinal complaints. It has been 
postulated that, the symptoms are produced because of a transient inability of thick bile to pass through the small openings between the septa, resulting in stasis and increased intraluminal pressure of the gallbladder,7. USG examination of the gallbladder is usually sufficient to diagnose MSG, although other modalities such as computed tomography, magnetic resonance cholangiopancreatography (MRCP) and endoscopic retrograde cholangiopancreatography have been described to establish the diagnosis ${ }^{8}$. In patients with multiseptate gallbladder, sonography demonstrates multiple linear, fine echogenic bands without acoustic shadowing or septa crossing the lumen of the gallbladder, giving the organ a honeycomb appearance ${ }^{9}$. MSG, especially in childhood, may coexist with choledochal cysts ${ }^{10}$, thus a detailed radiological examination of the hepatobiliary system is needed to exclude a choledochal or pancreatic pathology. Cholelithiasis ${ }^{11,12}$ and acute acalculous cholecystitis $^{13}$ are the other two complications of MSG. Except for biliary tract anomalies, a relationship between cholangiocarcinoma and MSG is also known ${ }^{14,15}$. Regarding the potential associated anomalies and risk of cancer, regular follow-up is undoubtedly substantial in an asymptomatic patient with MSG. On sonographic examination, desquamated gallbladder mucosa and the hyperplastic cholecystoses must be considered in the differential diagnosis. Desquamated gallbladder mucosa is seen as multiple linear echoes in the gallbladder lumen which do not arise from the wall of the gallbladder and the clinical setting is compatible with acute cholecystitis ${ }^{16}$. The appearance of polypoid cholesterolosis and adenomyomatosis may mimic multiseptate gallbladder, but there is no bridging of the gallbladder lumen by the cyst-like Rokitansky-Aschoff sinuses or polypoid bulbous echoes. A hydatid cyst should also be considered in the differential diagnosis. Cholecystectomy provides the relief of the symptoms in symptomatic patients with MSG17, while nonoperative management with regular follow-up is reasonable in the absence of the symptoms attributable to the MSG or an

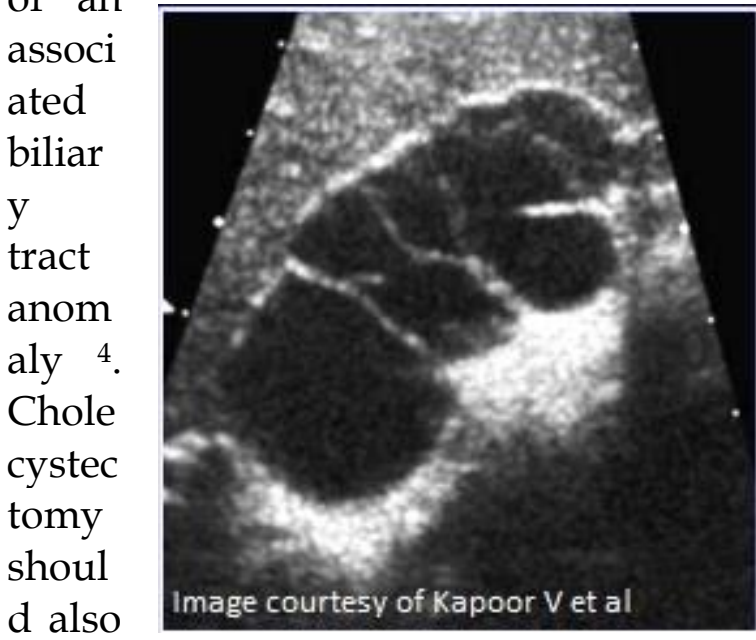

be considered in elderly, asymptomatic patients in whom MSG is incidentally discovered, due to the possibility of undetected carcinoma of the gallbladder $^{18}$. In conclusion, MSG, although rare, should always be considered in the differential diagnosis of children presenting with recurrent attacks of abdominal pain. Recurrent abdominal pain in childhood due to gallbladder is often misinterpreted as being due to intestinal and genitourinary etiologies. Cholelithiasis and cholecystitis are very rare before puberty and, if present, they are mostly 
related to bacterial and parasitic infections, hemolytic conditions and chronic gastrointestinal diseases ${ }^{19}$. We think that the cause of recurrent abdominal pain in our patient was the mechanical effect of septa impairing bile flow. As these septa do not contain muscle fibers, difficulty in bile flow is a consequence of the impaired motility of the gallbladder. Multiseptate gallbladder, although rare, should also be borne in mind among the etiologic factors of recurrent abdominal pain, and abdominal ultrasound should form part of the investigation.

\section{References}

1. HASLAM RHA, GAYLER BW, EBERT PA. Multiseptate Gallbladder: A Cause of Recurrent Abdominal Pain in Childhood. Am J Dis Child. 1966;112(6):600-603.

doi:10.1001/archpedi.1966.020901 50144021

2. Simon $\mathrm{M}$, Tandon BN. Multiseptate gallbladder. Radiology 1963;80:84-86

3. Bhagavan BS, Amin PB, Land AS, Weinberg T. Multiseptate gallbladder: embryogenetic hypotheses. Arch Pathol 1970;89:382-85.

4. Wanaguru D, Jiwane A, Day AS, Adams S. Multiseptate Gallbladder in an Asymptomatic Child. Case Rep Gastrointest Med. 2011;2011:470658.

A. Ozgen, D. Akata, and M. N. Ozmen, "Case report: multiseptate gallbladder," Turkish Journal of Diagnostic
Interventional Radiology, vol. 5, p. 496, 1999.

5. Strauss S, Starinsky R, Alon Z. Partial multiseptate gallbladder: sonographic apperance. J Ultrasound Med 1993;12:201-03.

6. Demirpolat G, Duygulu G, Tamsel S. Multiseptate gallbladder in a child with recurrent abdominal pain. Diagn Interv Radiol 2010;16:306-07.

7. Karaca $T$, Yoldas $\mathrm{O}$, Bilgin BC, Bilgin S, Evcik E, Ozen S. Diagnosis and Treatment of Multiseptate Gallbladder with Recurrent Abdominal Pain. Case Rep Med vol. 2011;2011:162853.

8. Lev-Toaff AS, Friedman AC, Rindsberg SN, et al. Multiseptate gallbladder: incidental diagnosis on sonography. AJR Am J Roentgenol 1987; 148:1119.

9. Pery M, Kaftori JK, Marvan H. Ultrasonographic appearance of multiseptate gallbladder: report of a case with coexisting choledochal cyst. J Clin Ultrasound 1985;13:570-73.

10. Erdogmus B, Yazici B, Ozdere BA, Akcan Y. Clinical and ultrasonographical findings in patients with multiseptate gallbladder. Tohoku J Exp Med 2004;204:215-19.

11. Croce EJ. The multiseptate gallbladder. Arch Surg 1973;107:104-05.

12. Erdogmus B, Yazici BB, Safak AA, Ozdere BA. Multiseptate gallbladder with acute acalculous cholecystitis. J Clin Ultrasound 2004;32:423-24. 
13. Ono S, Sakai K, Kimura O, Iwai N. Development of bile duct cancer in a 26-year-old man after resection of infantile choledochal cyst. J Pediatr Surg 2008;43:17-19.

14. Nakazawa $T$, Ohara $H$, Sano $H$, et al. Multiseptate gallbladder: diagnostic value of MR cholangiography ultrasonography. and Imaging 2004;29:691-93.

15. Wales LR. Desquamated gallbladder mucosa: unusual sign of cholecystitis. AJR Am J Roentgenol 1982; 139:810-811.

19. ediatr Surg 1992; 27:1560-1562.
16. Bigg RL. Multiseptate gallbladder. Arch Surg 1964;88:501-02.

17. E. Y. Rivera-Troche, M. G. Hartwig, and S. N. Vaslef, "Multiseptate gallbladder," Journal of Gastrointestinal Surgery, vol. 13, no. 9, pp. 1741-1743, 2009.

18. Esper E, Kaufman DB, Crary GS, et al. Septate gallbladder with cholelithiasis: a cause of chronic abdominal pain in a 6-year-old child. 